\title{
Quantum numerical control of atom-ion collision in intense laser field ${ }^{\text {a) }}$ )
}

\author{
Quan-Fang Wang*,1, c) \\ ${ }^{1}$ Mechanics and Automation Engineering, Chinese University of Hong Kong, N.T., \\ Hong Kong
}

In this work, $\mathrm{p}+\mathrm{H}$ (proton hydrogen) atom-ion collision is considered as a quantum control objective through solving a quantum system described by time dependent Schrödinger equation. For different internuclear distances, the colliding particles are controlled via applying an appropriate intense laser field. It means that one can control certain particle collision in the viewpoint of quantum mechanics at the atomic scale. It is possible to achieve the quantum control of particular colliding process theoretically and computationally, although the realization for poly-particles collision are fairly inadequate. Furthermore, the enhanced ionization rate reaching maximum at a critical internuclear distance is also surveyed together in this way.

PACS numbers: 50.20.Hv, 02.30.Yy, 03.65.Yz, 46.15.Cc

Keywords: quantum optima control, atom-ion collision, computational approach

a) * Corresponding author

b) Previous work "Quantum controlling for nuclear collision" see 239th American Chemistry Society National Meeting \& Exposition, 2010, poster.

c)Electronic mail: quanfangwang@hotmail.com;qfwang@mae.cuhk.edu. 


\section{INTRODUCTION}

Quantum physics, nuclear physics and particle physics had made significant progress in the last half of century ${ }^{1-4}$. The historic milestones include electron-positron collision and a mount of interdisciplinary breakthroughs. Physicists and chemists established the standard model as the precise theory of high-energy particle colliders. For example, the annihilation of an electron-positron pair to produce a fermion anti-fermion pair, and so forth. With the completeness of laboratory equipments, such as LHC, RHIC, PHENIX, SYS, ALICE, nuclear collision is well considered for multiplicity purposes. The established colliders provided us the feasibility in the particle physics to produce and detect particles. It is convenient to find the collision photos of proton-proton, electron-positron at CERN.

The raising question is how to control the nuclear collision for many body problems? That is how to control nuclear events to be represented as processing from ground initial state to final target state under the external control forcing? for instants, laser pulse beam, atomic laser etc.

In order to find the answer, the goal of our work is laying on controlling of certain quantum collision. Actually, with tremendously studying of quantum control at molecules and atoms scale, control of nuclear events is becoming the essential part in the forthcoming control mission in a variety of fields. As an attempt research on controlling of nuclear collision, it is aim is to proceed theoretical and computational controlling in real scale of physical constants and variables perfectly.

As is well known that a varieties of collisions are studied in theoretical and experimental researches for molecules and atoms. Most complicated works are concerning with ionizations of molecules and atoms, dissociated ionizations by intense laser field ${ }^{5-7}$. In the standpoint of physics, the electrons ionization rates are quite influenced by the inter-nuclear distance. At the so-called critical inter-nuclear distance, where the ionization rate can reach a maximum. $\mathrm{p}+\mathrm{H}$ collisions is selected as the phenomena to observe the sensitivity to inter-nuclear distance with the changing of intense laser field. As to the general nuclear collisions of poly particles, their process are too tough to be controlled owing to the uncertainties of the motion for each particle. For example, the large number of particles collision, the proton-proton collisions taken placed in LHC dated on March 30, 2010. It would be difficult to ensure the motion of per particles within the collision under external forces. Fortunately, for specified 
collision process (e.g. atom-ion $\mathrm{p}+\mathrm{H}$ ), one can easily clarified the motion of each particle in its collision. Therefore, it is adequate for us to survey those kinds of collision, and regard it as our target to be controlled.

The purpose of this article is to utilize the intense laser field in tracking the collision process of $\mathrm{p}+\mathrm{H}$ atom-ion. Further, at a critical internuclear distance, it should be found that ionization rate as it reached maximum. Our motivation is to simulate the process of $\mathrm{p}+\mathrm{H}$ collision with laser controller for attaining the experimental conclusion in physics field.

This paper is organized by following sections. Section 2 is to show the basic concepts of quantum system for $\mathrm{p}+\mathrm{H}$ collision in Hilbert space, which is defined by time-dependent Schrödinger equation. Section 3 is to set the control theory for collision of $\mathrm{p}+\mathrm{H}$ particles

system. Section 4 is to address the computational demonstration results. Section 5 contains the concluding remark.

\section{II. $\mathbf{P}+\mathrm{H}$ COLLISION SYSTEM}

In ultra energy collision, two particles collision for atom-ion $\mathrm{p}+\mathrm{H}$ is occurred in two spatial dimensions in classical viewpoint. Suppose $\mathbf{x}=\left(x_{1}, x_{2}\right)$ is the variable of spatial space $\Omega$, which is the open bounded set of $\mathbf{R}^{2}$.

Denote $Q=\Omega \times(0, T)$, then $(\mathbf{x}, t) \in Q$. Let $q$ be the charge of electron, and $m_{e}, m_{p}$ be mass of electron and proton, respectively. By the investigation of collision of $\mathrm{p}+\mathrm{H}$ atom-ion, it is pretty easy to describe the quantum system in the form of time-depended Schrödinger system.

$$
\left\{\begin{array}{l}
i \hbar \frac{\partial \psi}{\partial t}+\frac{1}{2 \mu} \frac{\partial^{2} \psi}{\partial \mathbf{x}^{2}}+V(\mathbf{x}, R) \psi=q\left(x_{1}+x_{2}\right) E(t) \psi \\
\psi(0)=\psi_{0}
\end{array}\right.
$$

Here in (1), wave function $\psi$ denotes the electron motion, $\mathbf{x}$ is the electron coordinate with respective to the center of mass of the two protons. Let

$$
\mu=\frac{2 m_{e} m_{p}}{m_{e}+2 m_{p}}, \quad q=1+\frac{m_{e}}{m_{e}+2 m_{p}} .
$$


and $V(\mathbf{x}, R)$ is the external energy potential given by

$$
\begin{aligned}
V(\mathbf{x}, R)= & \frac{-1}{\left[\left(x_{1}-R / 2\right)^{2}+1\right]^{1 / 2}}+\frac{-1}{\left[\left(x_{1}+R / 2\right)^{2}+1\right]^{1 / 2}} \\
& +\frac{-1}{\left[\left(x_{2}-R / 2\right)^{2}+1\right]^{1 / 2}}+\frac{-1}{\left[\left(x_{2}+R / 2\right)^{2}+1\right]^{1 / 2}} .
\end{aligned}
$$

Here in $(2), V(\mathbf{x}, R)$ is a Coulomb interaction between the electron and two protons. $R(t)$ is assumed as the internuclear distance at time $t$. In (1), the variable

$$
E(t)=E_{0} U(t) \cos (\omega t)
$$

is the applied electronic field, where $E_{0}$ is the amplitude of the laser electronic field, and $\omega$ is the laser frequency. $U(t)$ denotes the envelop function of laser pulse (taking $U(t)=1$ in later experiment of Section IV). In the simulation, the electronic outside a given region $|\mathbf{x}|<d / 2$ ( $d$ is parameter) is considered to be ionized. Thus, the corresponding probability $P_{I}(t)$ is calculated by

$$
P_{I}=1-\int_{-d / 2}^{d / 2}|\phi(\mathbf{x}, t)|^{2} d \mathbf{x},
$$

where the region is taken to be 70 a.u. The ionization rate is given by

$$
\alpha(t)=\frac{1}{\delta t} \ln \left(\frac{1-P_{I}(t-\delta t / 2)}{1-P_{I}(t+\delta t / 2)}\right), \quad \delta t=\frac{T}{10} .
$$

In the framework of variational method, quantum optimal control theory (QOCT) will be applied to $\mathrm{p}+\mathrm{H}$ system (1). Introduce two Hilbert spaces $H=L^{2}(\Omega), V=H_{0}^{1}(\Omega)$ with usual norm and inner product see paper ${ }^{2}$. Notice that without confusion of notations $V$ and $V(\mathbf{x}, R)$. Thereby the embedding in Gelfand triple space $V \hookrightarrow H \hookrightarrow V^{\prime}$ are continuous, dense and compact. This provides us the possibility to utilize optimization procedure paper ${ }^{11}$ to give the appendix definitions in general manner.

Definition 1 Define weak solutions affiliated solution space by Hilbert space:

$$
W\left(0, T ; V, V^{\prime}\right)=\left\{\psi \mid \psi \in L^{2}(0, T ; V), \psi^{\prime} \in L^{2}\left(0, T ; V^{\prime}\right)\right\}
$$

Definition $2 A$ wave fucntion $\psi$ is called weak solution of (1), if $\psi \in W\left(0, T ; V, V^{\prime}\right)$ and satisfy the weak form

$$
\begin{aligned}
& \int_{0}^{T} \int_{\Omega}\left[-i \hbar \frac{\partial \eta}{\partial t}-\frac{1}{2 \mu} \frac{\partial \psi}{\partial \mathbf{x}} \frac{\partial \eta}{\partial \mathbf{x}}+V(\mathbf{x}, R) \psi \eta+q\left(x_{1}+x_{2}\right) E(t) \eta\right] d \mathbf{x} d t \\
= & \int_{\Omega} i \hbar \psi^{0} \eta(0) d \mathbf{x},
\end{aligned}
$$

for all $\eta \in C^{1}(0, T ; V)$ and $\eta(T)=0$, a.e. $t \in[0, T]$. 


\section{OPTIMAL QUANTUM CONTROL}

Let $E(t)$ be regarded as the control function in $\mathrm{p}+\mathrm{H}$ quantum system (1). Suppose $\mathcal{U}=L^{2}(0, T)$ is the space of control variable $E(t)$, and $\mathcal{U}_{a d}$ be a closed and convex admissible set of $\mathcal{U}$. The cost criteria associated with (1) is given by

$$
J(E)=\epsilon_{1}\left(1-\frac{1}{2} \int_{0}^{T} \int_{\Omega} \bar{\psi}_{f}(E) \psi_{\text {target }} d \mathbf{x} d t\right)+\epsilon_{2} \int_{0}^{T} E(t)^{2} d t,
$$

for all $E(t) \in \mathcal{U}_{a d}$. Here in (6) $\psi_{\text {target }} \in V$ is target state and $\bar{\psi}_{f}(E)$ is the conjugate of observed final state. The parameters $\epsilon_{1}, \epsilon_{2}$ are weighted coefficients for balancing the values of inherent and running cost. The choice of the form of performance index (6) is different than the usual one given by $\frac{1}{2}\left\|\psi_{f}(E)-\psi_{\text {target }}\right\|^{2}$ as in most of literatures. It is based upon the reason of norm regularity $\left\|\psi_{f}(E)\right\|=\left\|\psi_{\text {target }}\right\|=1$ for wave function of quantum system (1).

Obviously, using weak form (5), by analogously manipulating as in Paper ${ }^{8,10,11}$ to prove the resultant Theorems. Omit their proofs for limited paper length.

Theorem 1 If $\psi_{0} \in V$, then there exists a unique weak solution $\psi$ for $p+H$ quantum system (1).

Theorem 2 If $\psi_{0} \in V$, then at least one quantum optimal control exists for cost function (6) subject to $p+H$ system (1).

Theorem 3 Let above assumptions are satisfied, then the quantum optimal control $E^{*}(t)$ for $p+H$ quantum system (1) subject to cost function (6) is characterized by the following simultaneously optimality system:

$$
\begin{aligned}
& \left\{\begin{array}{l}
i \hbar \frac{\partial \psi}{\partial t}+\frac{1}{2 \mu} \frac{\partial^{2} \psi}{\partial \mathbf{x}^{2}}+V(\mathbf{x}, R) \psi=q\left(x_{1}+x_{2}\right) E^{*}(t) \psi \\
\psi(0)=\psi_{0}
\end{array}\right. \\
& \left\{\begin{array}{l}
i \hbar \frac{\partial p}{\partial t}+\frac{1}{2 \mu} \frac{\partial^{2} p}{\partial \mathbf{x}^{2}}+V(\mathbf{x}, R) \psi=0 \\
i p(T)=\psi_{f}-\psi_{\text {target }}
\end{array}\right. \\
& \left(E^{*}, E-E^{*}\right)_{\mathcal{U}}+\int_{Q} p\left(E^{*}\right)\left(E-E^{*}\right) d \mathbf{x} d t \geq 0
\end{aligned}
$$

for all $E \in \mathcal{U}_{a d}$, where $p \in W\left(0, T ; V, V^{\prime}\right)$ is solution of the adjoint system (8) corresponding to solution $\psi$ in the state system (7), respectively. Clearly, as is well known that the inequality (9) is called necessary optimality condition. 


\section{DEMONSTRATION EXPERIMENTS}

For physical needs, real scale of each physical constant is used as the gauge in numerical demonstration. Take two dimensional case spatial variable $x=\left(x_{1}, x_{2}\right) \in \mathbf{R}^{2}$. Set proton mass $M=1.6262158 \times 10^{27} \mathrm{~kg}$. The electron mass $m=9.10938188 \times 10^{-31} \mathrm{~kg}$, its charge $e=-1.60218 \times 10^{-19} c$. Then $\mu=1.82088 \times 10^{30} \mathrm{~kg}, q=1.00027 \mathrm{e}$ and $\hbar=1.05457 \times 10^{-34}$ Js. Let domain $\Omega=(-20,20) \times(-20,20)$, start time $t_{0}=0.0 \mathrm{fs}$, final time $T=3500 \mathrm{fs}$, where fs denote femtosecond. Set $E_{0}=1.0, \omega=100.0$ and intense laser field $E(t)=E_{0} \cos (\omega t)$. Then initial laser input $E_{0}(t)=9.0 \times 10^{14} E(t)$, their graphics see Figure 1 . The internuclear

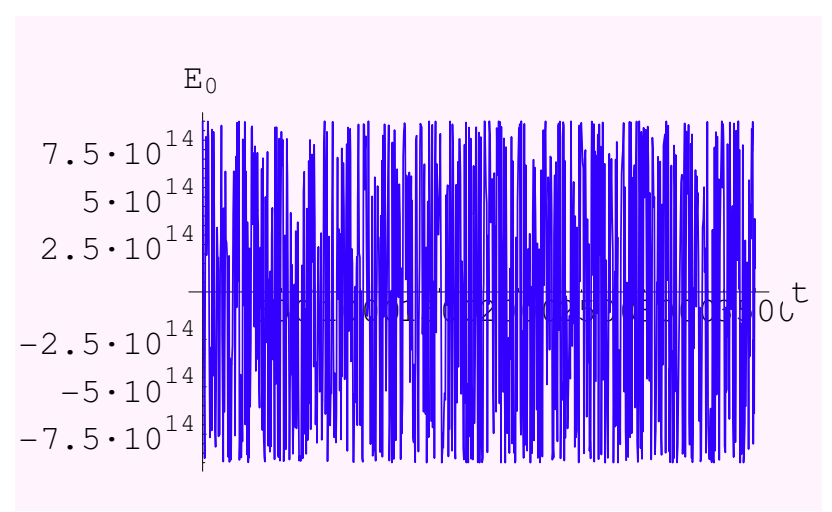

FIG. 1. Initial intense laser field $E_{0}(t), t \in[0,3500]$.

distance is also plotted in Figure 2. Notice that

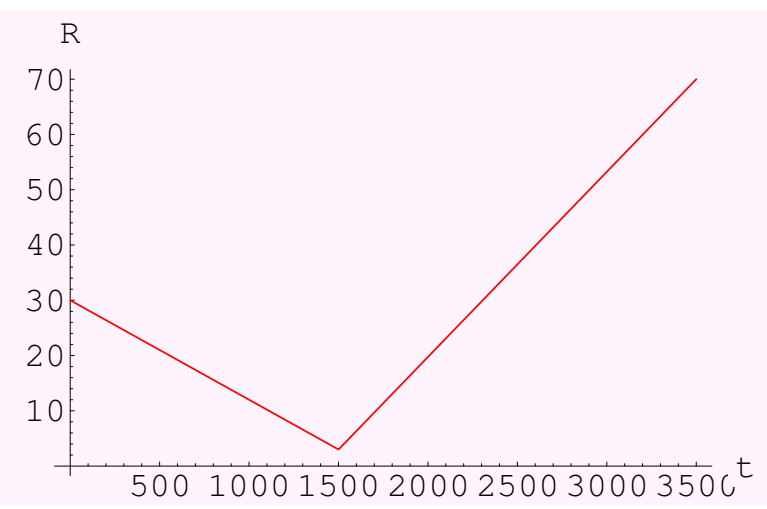

FIG. 2. Internuclear distance $R(t), t \in[0,3500]$.

$$
\begin{aligned}
& R(t)=-0.018 t+30, \quad 0 \leq t \leq 1500 \\
& R(t)=0.0335 t-47.25, \quad 1500 \leq t \leq 3500
\end{aligned}
$$


is used in following calculations. The initial state is given by

$$
\phi_{0}=1.0 \times 10^{-40} \exp \left[-\left(\frac{x_{1}}{10}\right)^{2}-\left(\frac{x_{2}}{10}\right)^{2}\right]
$$

and desired state function is given by $\psi_{\text {target }}=0.01 \psi_{0}$.

The total kinetic energy of fragments is approximately equal to $E_{k}=q_{1} q_{2} / R, q_{1}=$ $+q, q_{2}=-q$ are the charge of the two fragments, respectively. $R$ is the internuclear distance.

Set coefficient $E_{k 0}=0.1, \omega_{0}=2.0$, and initial internuclear distance $R_{0}=30.0, R_{T}=70.0$. Let us define

$$
x_{R}=\exp \left(\frac{R-R_{0}}{2 \omega_{0}}-i \sqrt{M E_{k 0}} R\right) .
$$

Thus real initial input $\psi_{0}=x_{R} \phi_{0}$, its graphics see Figure 3. Using the updated optimization
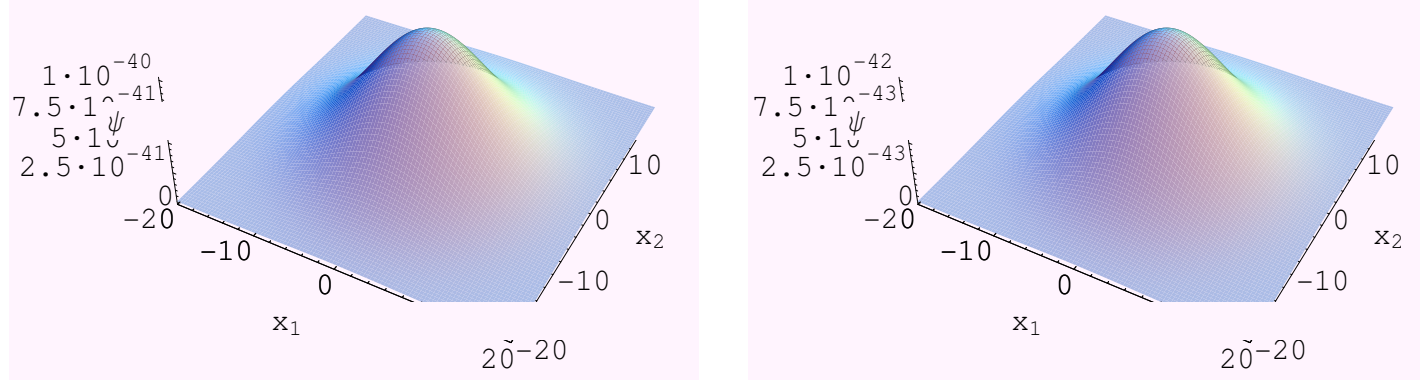

FIG. 3. initial ground state $\psi(0)$, desired target state $\psi(T), \mathbf{x} \in(-20,20) \times(-20 \times 20)$.

algorithm consist of conjugate gradient method and finite element approach as in article ${ }^{9}$, by limited iteration steps (stop at $n=4$ ), the collision states $\psi(t)$ transferred in Figure 4 for different time $t$.

In the mean time, their contour plots refer Figure 5 for corresponding changes of $\psi(t)$ respect to $t$.

Let us define nuclear distribution function by

$$
f(R)=\int|\psi(\mathbf{x}, R, t)|^{2} d \mathbf{x} .
$$

In two dimension $\mathbf{x}=\left(x_{1}, x_{2}\right), f(R)$ changed with time point at $t=0,1500,3500$ fs. Their plots graphics are shown in following Figure 6, respectively.

The corresponding contour plots also can be cited Figure 7. 


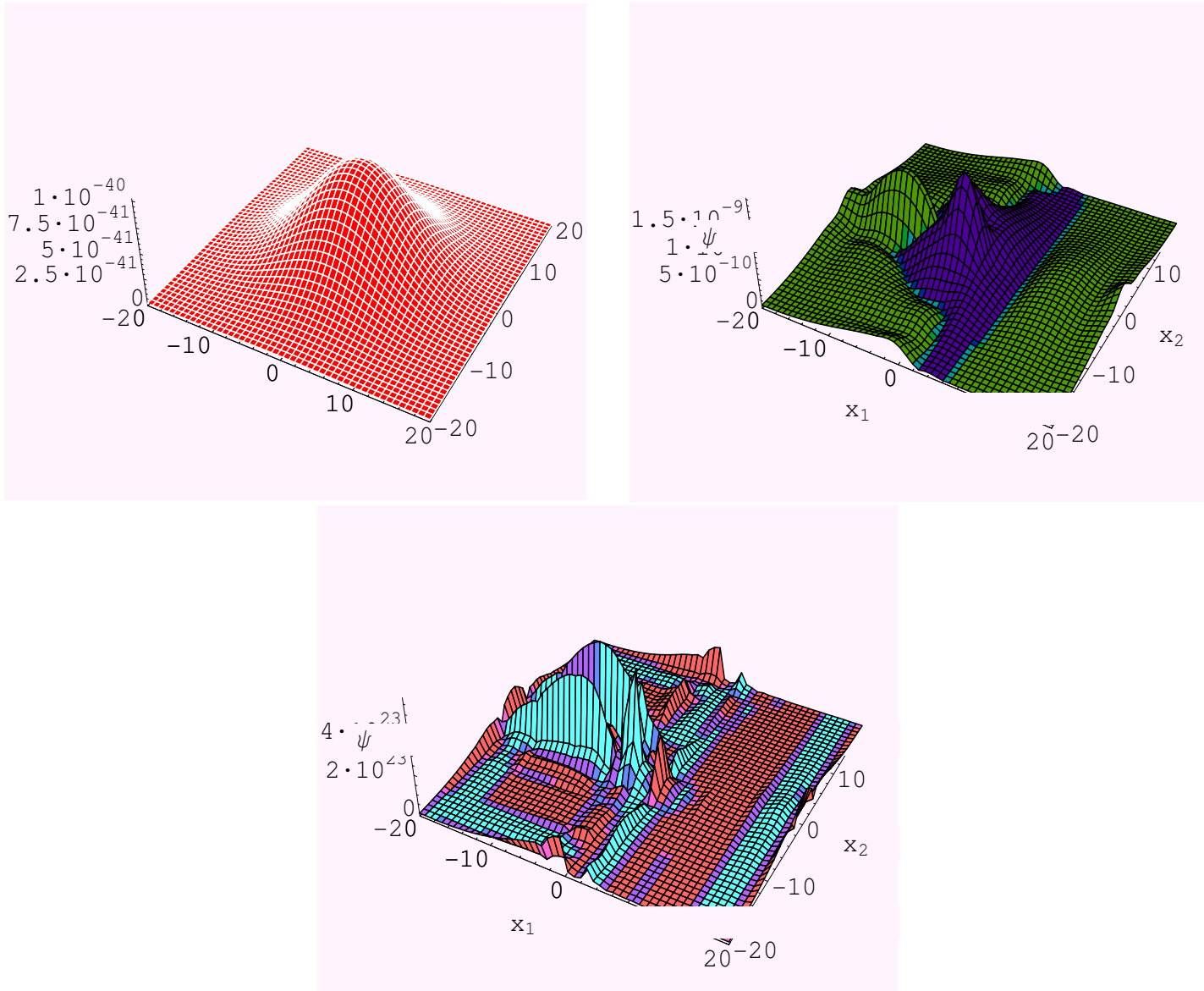

FIG. 4. $\psi(t)$ iteration at $t=0,1500,3500$ fs.

It is clear to expose the status of two particle before and after their collision in above graphics analysis. For more details, in the direction according two axes $x_{1}, x_{2}$, their collision occurred with plots of $f(R)$ in Figure 8 .

One can find in Figure 8, the nuclear distribution function $f(R)$, wavepackage centeris on the left hand of $x_{1}$ and $x_{2}$ domain. More electrons distributed in the left potential well. The ionization rate is higher for the negative laser electronic filed than those for the positive laser electronic field. The results agree with the experimental results for $\mathrm{p}+\mathrm{H}$ atom-ion collision, see paper ${ }^{7}$ Fig.4 and Fig. 5.

Via a limited iteration, optimal control (external input, e.g. laser pulse) is calculated as

$$
E^{*}(t)=179686.0-2.1005 \times 10^{8} \sin (100 t) .
$$

Moreover, the error between $E^{*}(t)$ and $E_{0}(t)$ is also obtained:

$$
E^{*}(t)-E_{0}(t)=-8658.89+14.2152 \cos (100 t) .
$$



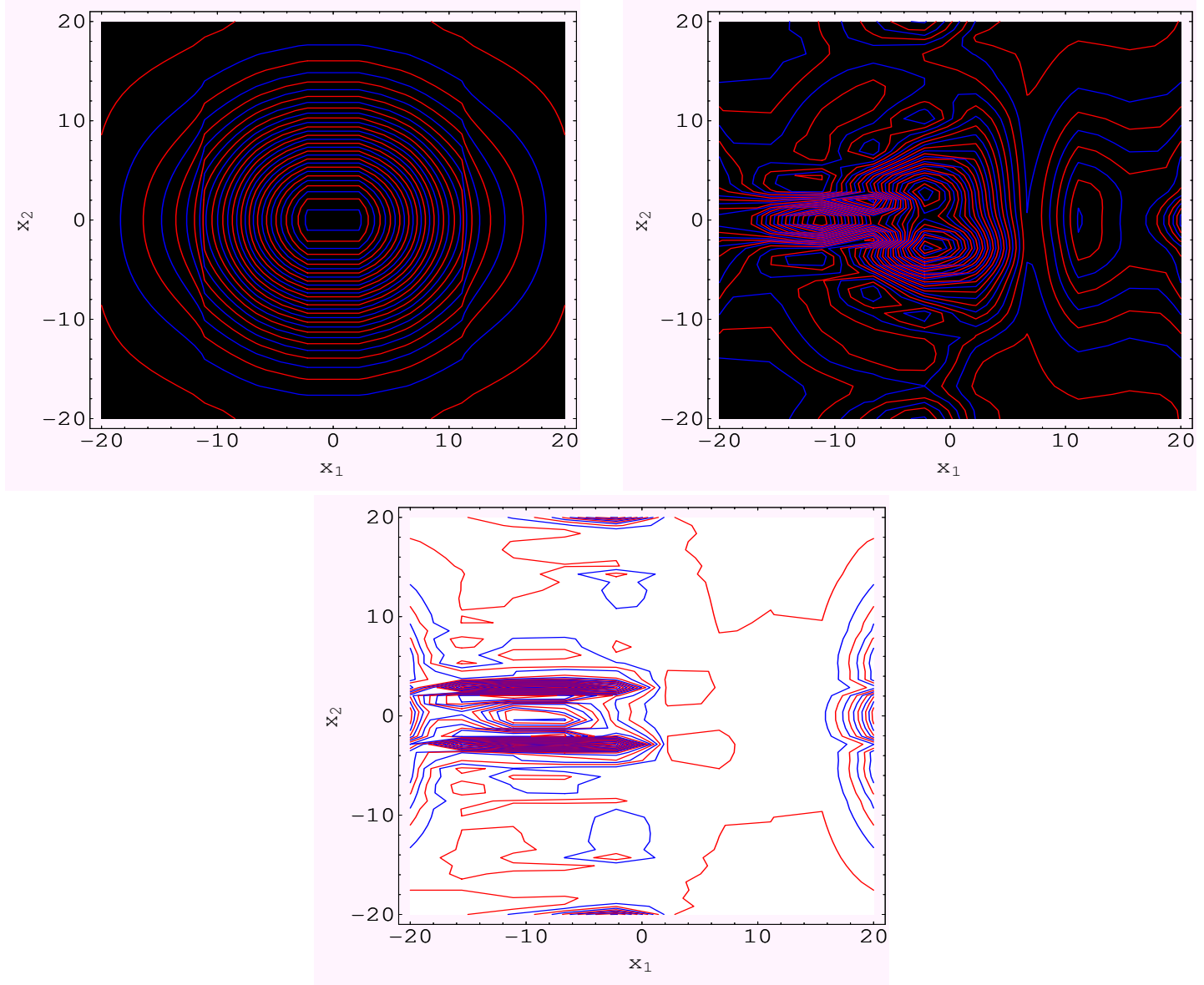

FIG. 5. Contour plots of $\psi(t)$ at $t=0,1500,3500$ fs.

The optimal control function $E^{*}(t)$, the cost functions iteration $J(E)$, and their error value $e J(E)$ are shown in Figures 9, respectively.

Surely, the collision make cost function increasing with the iterations and time $t$. By the reason of strong external force input described by (11), the energy is not conserved in the entire colliding. Here are their values at each iteration:

$$
\begin{array}{ll}
J_{1}=8.84066 \times 10^{30}, & J_{2}=1.43269 \times 10^{33}, \\
J_{3}=1.43269 \times 10^{33}, & J_{4}=1.43269 \times 10^{33} .
\end{array}
$$

Error values of cost function in each iteration:

$$
\begin{array}{ll}
e J_{1}=8.84066 \times 10^{30}, \quad e J_{2}=1.42385 \times 10^{33}, \\
e J_{3}=3.02162 \times 10^{25}, \quad e J_{4}=0 .
\end{array}
$$

The possibility ionization function $P_{I}(t)$ in (3), the ionization rate $\alpha(t)$ in (4) and distribution function $f(R)$ values in (10) are executed in Figure 10. 

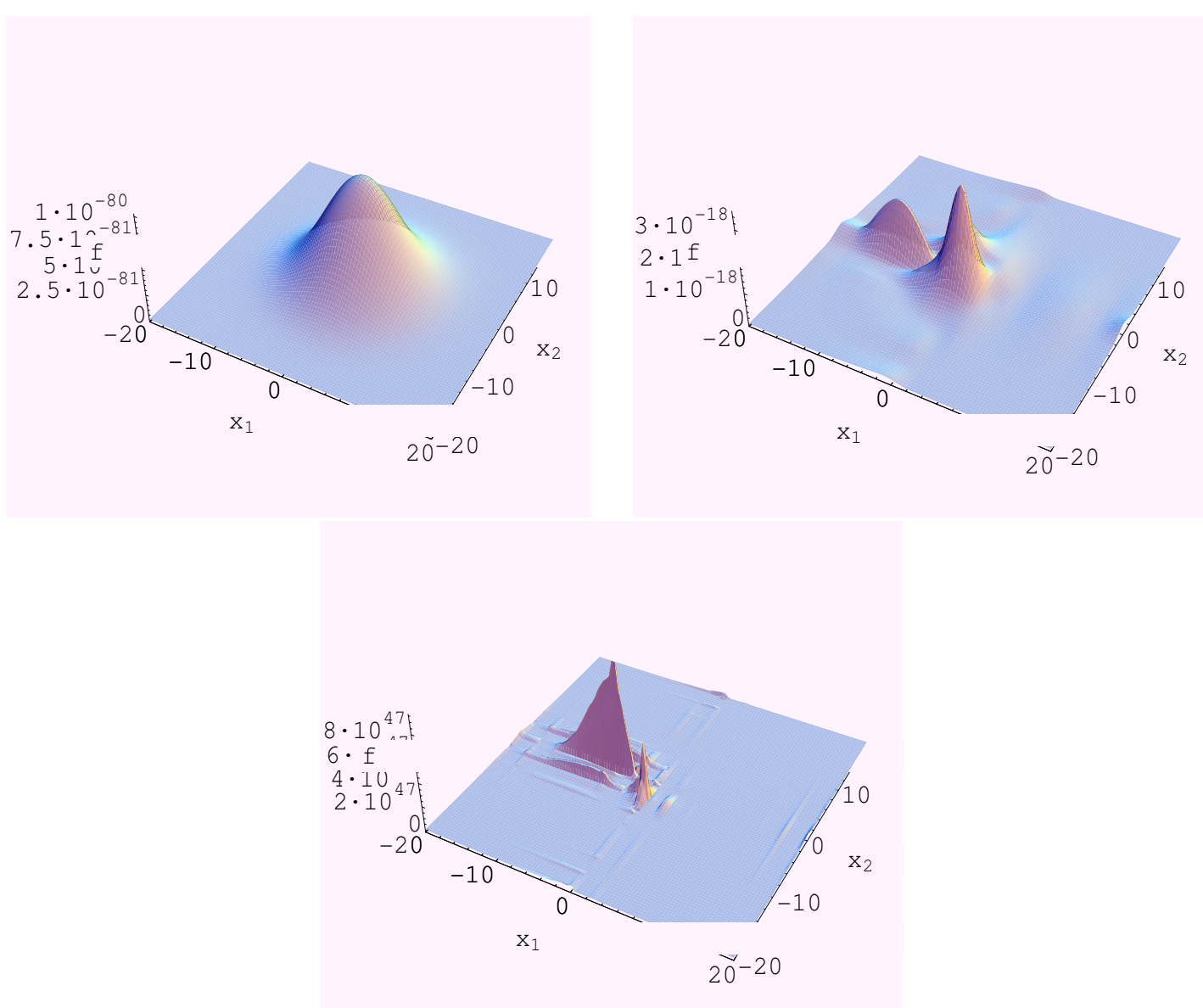

FIG. 6. Wave package $f(R)$ at $t=0,1500,3500$ fs.

Finally, the computed optimality value

$$
J\left(E^{*}\right)=1.43269 \times 10^{33}
$$

and minimization cost error $e J\left(E^{*}\right)=0$. The error of control function $e E$, energy function $E_{k}$ given by Figure 11. Through all calculation, the total used CPU time 12592 second, and maximum memory used is 110498200 bytes.

By the comparison with literatures and reported papers, the obtained results are in accordance with those conclusion in real physical experiments, refer papers ${ }^{5-7}$. Consequently, quantum control approach can be used to illuminate the coherence with other fields, and interpret each other efficiently. 

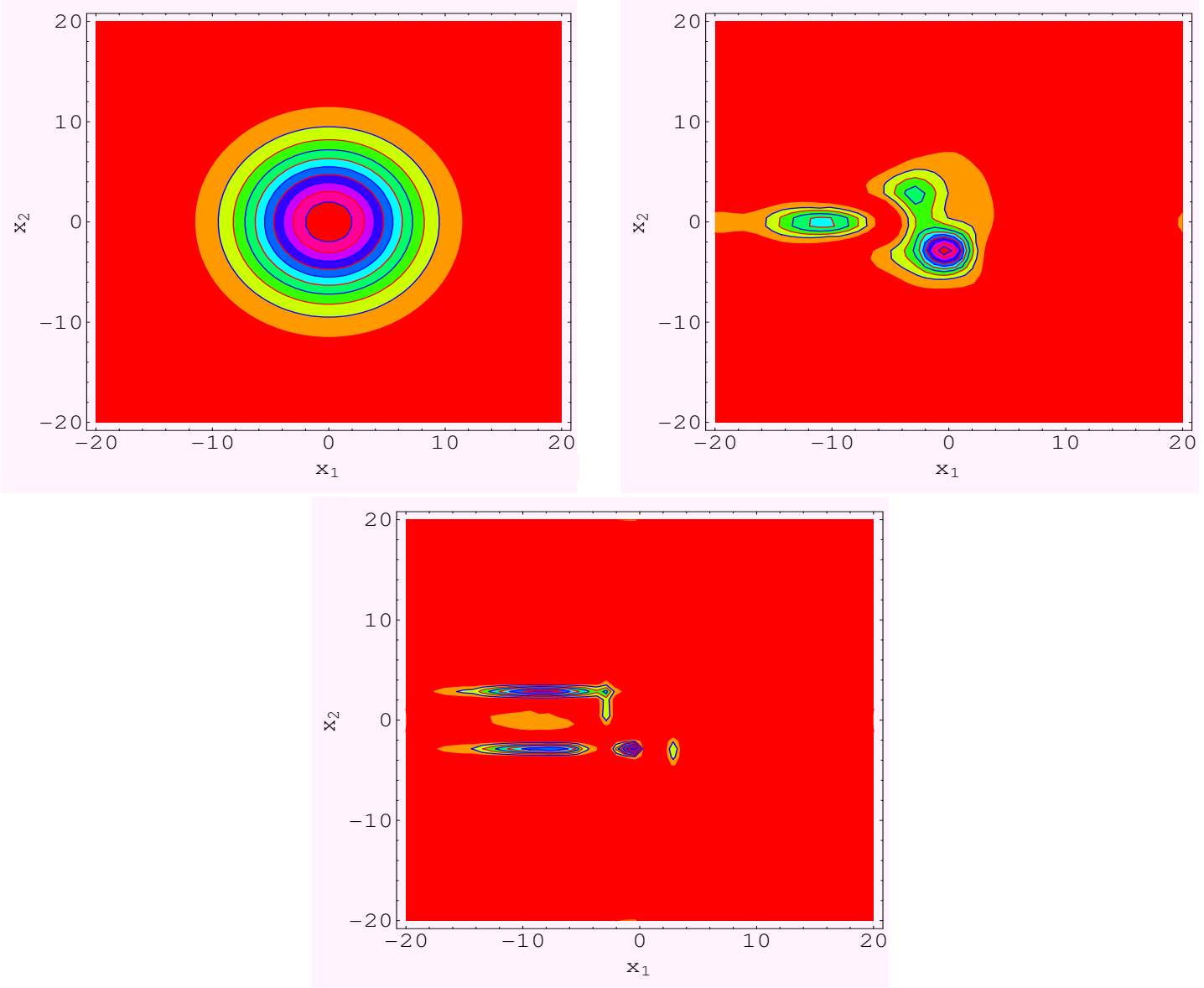

FIG. 7. Contour plots of $f(R)$ at $t=0,1500,3500$ fs.

\section{CONCLUSIONS}

This work presented the quantum control of $\mathrm{p}+\mathrm{H}$ nuclear collision problem. Especially, for atom-ion collision, theoretical and computational approach is studied for two-dimension case. By the demonstrated simulation results, it is easily found that the energy and states are changed together with the inter-nuclear distance, there exists a critical inter-nuclear distance, which is sensitive to ionization rates and other physical quantities. Resultant theoretic and computational conclusions are quite consistent with that in literatures ${ }^{5-7}$.

As perspective, it would be a promising direction to solve the relevant problems by acting the intense laser field as control input with respect to quantum dynamics system in nuclear collision and reactions ${ }^{12}$. 

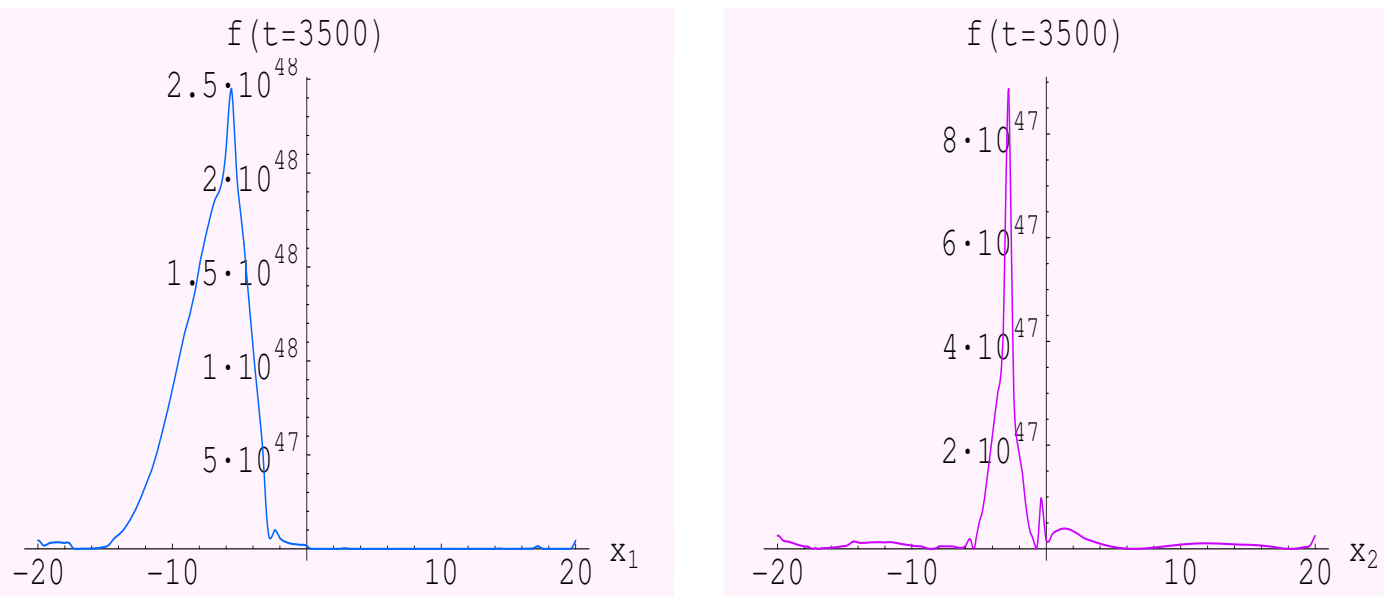

FIG. 8. Plot $f(R)$ in $x_{1}, x_{2}$ axes direction. $t=3500.0$ fs.

\section{ACKNOWLEDGMENTS}

The author would like to thank 239th American Chemistry Society National Meeting \& Exposition, for poster "Quantum controlling for nuclear collision" 92010.

\section{REFERENCES}

${ }^{1}$ E. U. Condon and P. M. Morse, "Quantum mechanics of collision processes I", Review of Modern Physics 3, 43, (1931).

${ }^{2}$ R. Dautary, and J. L. Lions, Mathematical Analysis and Numerical Methods for Science and Technology (Springer-Verlag, Berlin-Heidelberg-New York, 1992).

${ }^{3}$ P. M. Morse, "Quantum mechanics of collision processes II", Review of Modern Physics 4, 577, (1932).

${ }^{4}$ L. Eno and H. Rabitz, "Generalized sensitivity analysis in quantum collision theory", Journal of Chemical Physics 71(12), 4824, (1979).

${ }^{5}$ L. Feng, Y. Ho, "Energy absorption by charge-resonance-enhanced ionization during atomion collisions in an intense laser field", Physical Review A 58, 2332, (1998).

${ }^{6}$ L. Feng, Y. Ho, "The gain of kinetic energy of particles during atom-ion collisions in an intense laser field", Physical Letters A 251, 121, (1999).

${ }^{7}$ L. Feng, Y. Ho. "Quantum simulation of collinear $\mathrm{p}+\mathrm{H}$ collisions in an intense laser field", Physical Review A 61, 023407, (2000).

${ }^{8}$ Q. F. Wang, H. Rabitz, "Quantum optimal control for the Klein-Gordon-Schrödinger 

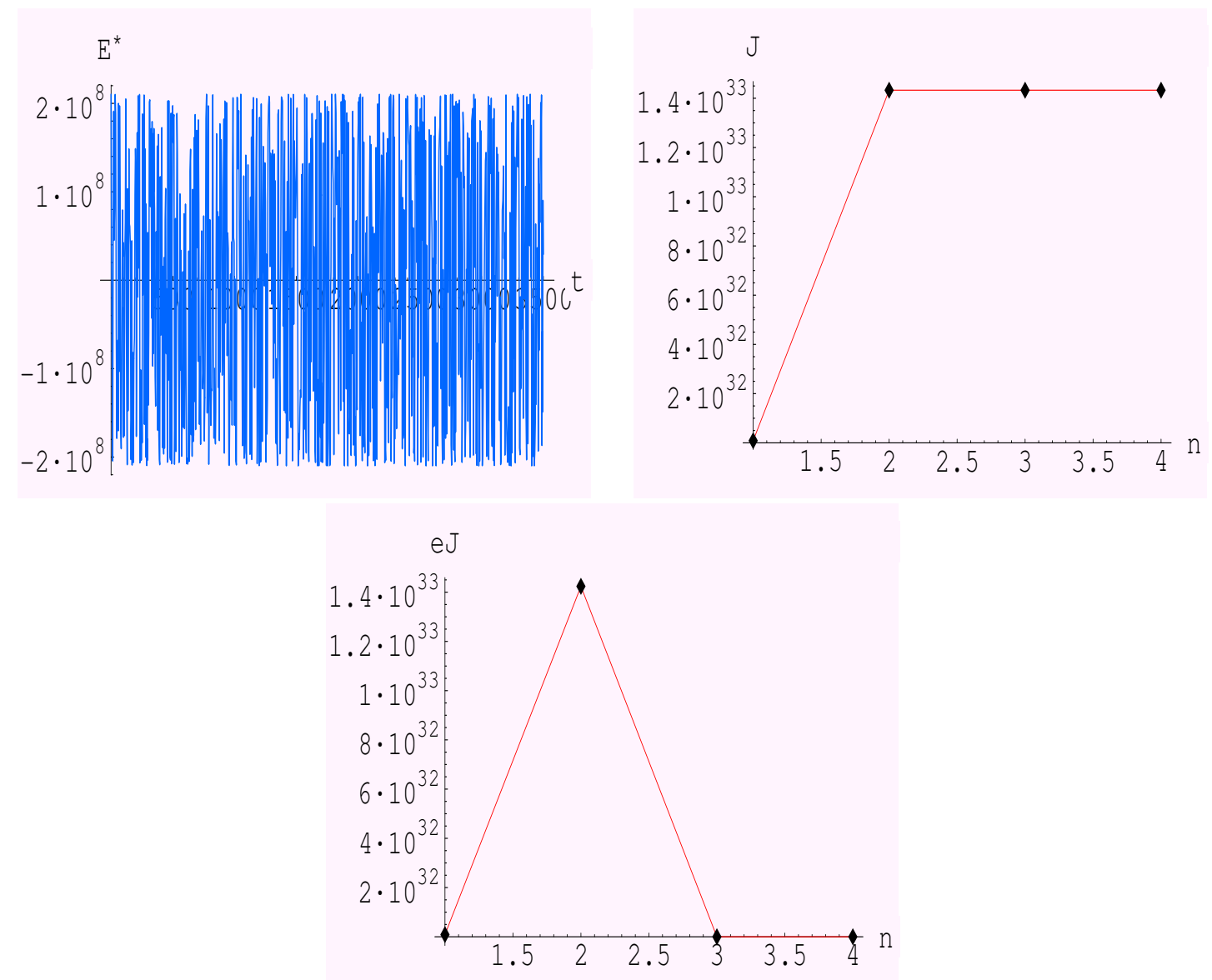

FIG. 9. $E^{*}(t)$ for $t \in[0,3500] . J(E), e J(E)$ at $n=1,2,3,4$.

dynamics system in the presence of disturbances and uncertainties", Gordon Research Conference Quantum Control of Light and Matter, RI, USA, Poster, (2007).

${ }^{9}$ Q. F. Wang, "Quantum controlling for nuclear collision", 239th American Chemistry Society National Meeting \& Exposition, poster, (2010).

${ }^{10}$ Q. F. Wang, V. P. Belavkin, "Quantum control for Bose-Einstein condensates", 4th International Scientific Conference on Physics and Control, Italy, (2009).

${ }^{11}$ Q. F. Wang, Optimal Control for Nonlinear Parabolic Distributed Parameter Systems: with numerical analysis (Lambert Academic Publishing, Germany, 2011).

${ }^{12}$ Q. F. Wang, Practical Application of Optimal Control Theory: computational approach (Lambert Academic Publishing, Germany, 2011). 

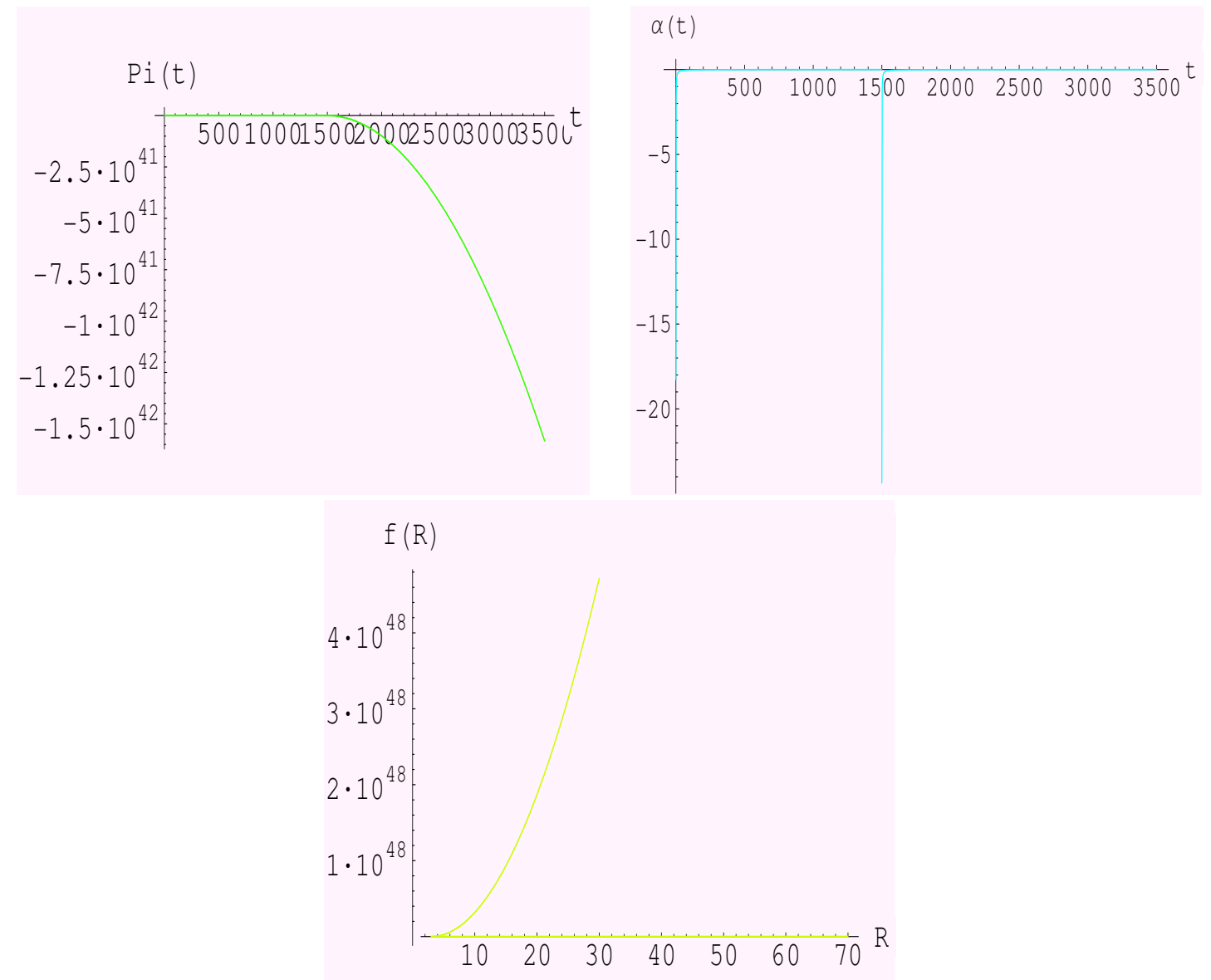

FIG. 10. $P_{I}(t), \alpha(t)$ for $t \in[0,3500], f(R)$ for $R \in[0,70]$.
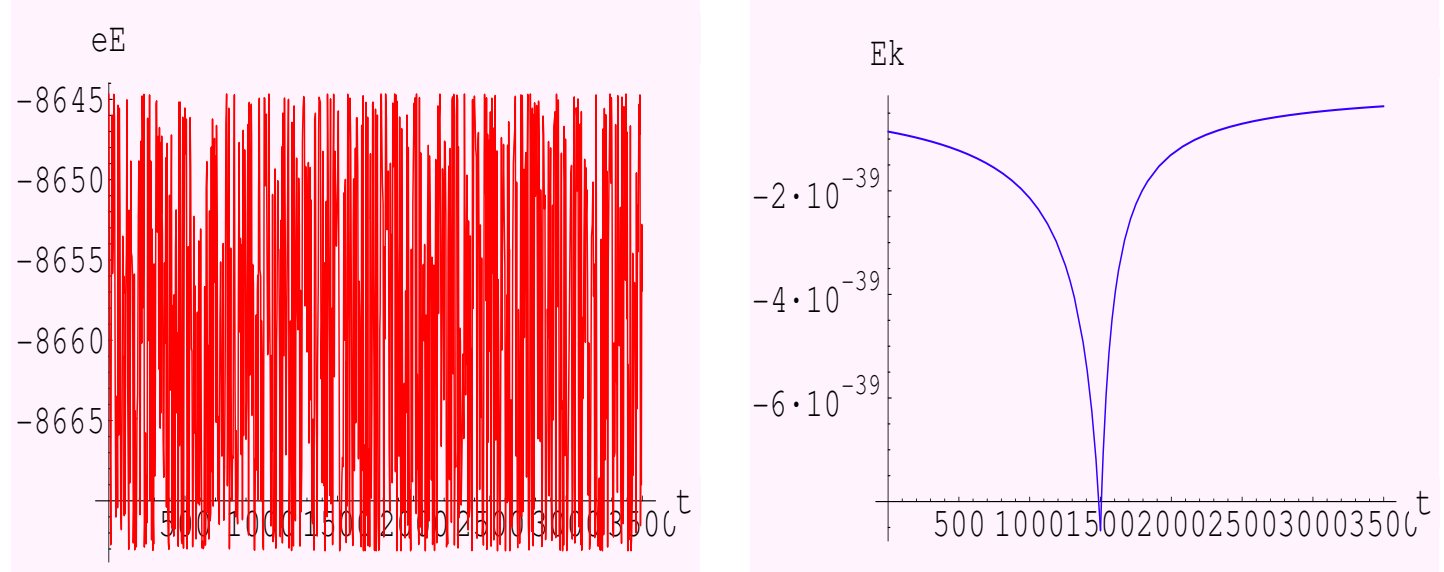

FIG. 11. $e E, E_{k}$ for $t \in[0,3500]$. 